\title{
Resonant Tunneling Through Two Discrete Energy States
}

\author{
N. C. van der Vaart, S. F. Godijn, Y. V. Nazarov, C. J. P. M. Harmans, and J. E. Mooij \\ Department of Applied Physics, Delft University of Technology, P.O. Box 5046, 2600 GA Delft, The Netherlands \\ L. W. Molenkamp* \\ Philips Research Laboratories, 5600 JA Eidenhoven, The Netherlands \\ C. T. Foxon ${ }^{\dagger}$ \\ Philips Research Laboratories, Redhill, Surrey RH15HA, United Kingdom
}

(Received 10 January 1995)

\begin{abstract}
We observed new type of Lorentzian-shaped resonances in the current through two coupled quantum dots with tunable barriers. We show that the resonances occur when the energy of two discrete states match. Their widths can be as small as $5 \mu \mathrm{eV}$ and are only determined by the lifetime of the discrete energy states, independent of the reservoir temperature. The achieved energy resolution makes it possible to observe a small asymmetric deviation from the Lorentzian line shape, which we attribute to inelastic tunnel processes.
\end{abstract}

PACS numbers: 71.50.+t, 73.20.Dx, 73.40.Gk

Artificially fabricated nanostructures, which contain only a few electrons, have proven to be excellent laboratories to study quantum size effects [1-7]. The electron wave nature gives rise to the formation of zero-dimensional (OD) states when electrons are confined in all three spatial directions in a quantum dot. Resonant tunneling through a OD state has been studied in detail by measuring the current through a quantum dot, weakly coupled to two electron reservoirs. Although this allows for a good characterization of the spectrum of the OD states, information about the intrinsic lifetime of the OD states or the tunnel mechanisms is partially masked by the temperature of the reservoirs [5].

This limitation can be removed when two dots are placed in series and the resonant tunneling current through two OD states is measured. In this case, transport is expected to be possible only when the energy of the two OD states matches. The current is therefore determined by the energy overlap of the two OD states [8]. Experiments on double quantum dots in vertical structures showed resonances, but the width could not be related to the intrinsic lifetime of OD states [9]. In this work, we use a tunable double quantum dot with well-developed OD states in each dot, and we exploit the Coulomb blockade of tunneling to control the number of electrons in the dots [10].

Using this technique, we observe sharp resonances when the energy of two OD states in two different dots matches. The shape of the peaks is Lorentzian, and the width is determined only by the lifetime of the OD states. Furthermore, we are able to distinguish between elastic and inelastic tunnel processes between the two dots and estimate a lower boundary for relaxation rates within a dot.

The double quantum dots used for this work are defined by metal gates on top of a GaAs/AlGaAs heterostructure with a two-dimensional electron gas (2DEG) $100 \mathrm{~nm}$ below the surface. The ungated $2 \mathrm{DEG}$ has a mobility of $2.3 \times 10^{6} \mathrm{~cm}^{2} / \mathrm{V} \mathrm{s}$ and an electron density of $1.9 \times$ $10^{15} \mathrm{~m}^{-2}$ at $4.2 \mathrm{~K}$. A scanning electron micrograph (SEM) of the gate geometry is shown in Fig. 1(a). Applying a negative voltage to all gates depletes the electron gas underneath them and forms two quantum dots in the 2DEG. Current can flow from the large electron reservoir on the left via the three tunnel barriers induced by the gate pairs $1-F, 2-F$, and $3-F$ to the reservoir on the right. The transmission of each tunnel barrier can be controlled individually by the voltage on gates 1,2 , or 3 . A single quantum dot can be defined in the 2DEG by applying only a voltage to gates 1,2 , I, and $F$ (dot I) or to gates 2,3 , II, and $F$ (dot II). In this way, we can characterize the individual dots and compare their properties to those of the double dot. The experiments are performed at zero magnetic field in a dilution refrigerator with a base temperature below $2 \mathrm{mK}$.

The current-volage $(I-V)$ curve of a single quantum dot provides two clear signatures for the presence of both Coulomb blockade effects and OD states. The insets of Fig. 2 show the $I-V$ 's of dots I (upper inset) and II (lower inset). At low bias voltages $V$, the current through the dot is suppressed by the Coulomb blockade [10]. Increasing the bias voltage lifts the blockade. The current shows a stepwise increase: Each time when an additional OD state falls in the energy window $\mathrm{eV}$ set by the bias voltage $V$, an extra transport channel is opened and the current increases with a step [3-5]. From the spacing of the current steps we obtain the average spacing $\delta$ of the 0D states: $\delta_{\mathrm{I}}=125 \mu \mathrm{eV}$ for dot I (upper inset) and $\delta_{\text {II }}=225 \mu \mathrm{eV}$ for dot II (lower inset). The difference in these two energies reflects the different lithographic sizes of the two dots [see Fig. 1(a)]. Accounting for the depletion areas, we estimate that dot I has a diameter 

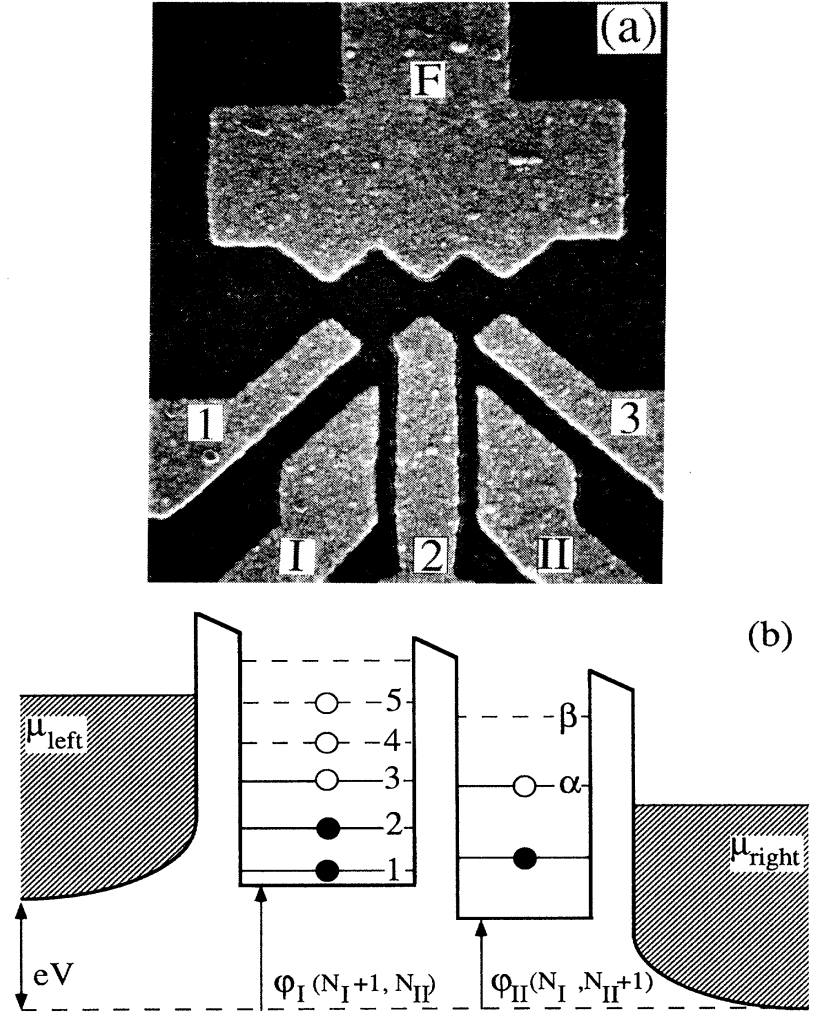

FIG. 1. (a) SEM micrograph of the double dot with lithographic dimensions of $320 \times 320 \mathrm{~nm}^{2}$ (left dot) and $280 \times$ $280 \mathrm{~nm}^{2}$ (right dot). (b) Schematic potential landscape of the double quantum dot, where $\mu_{\text {left }}$ and $\mu_{\text {right }}$ denote the electrochemical potentials of the left and right reservoirs and $V$ the bias voltage across the double dot. The $0 \mathrm{D}$ states in dot I are denoted by levels 1 to 5 and in dot II by levels $\alpha$ and $\beta$.

of $240 \mathrm{~nm}$ and contains about $N_{\mathrm{I}}=90$ electrons, while dot II has a diameter of $200 \mathrm{~nm}$ and contains roughly $N_{\text {II }}=60$ electrons. Using the Fermi energy $E_{F}$ at bulk density, we estimate $\delta_{\mathrm{I}} \approx 2 E_{F} / N_{\mathrm{I}}=150 \mu \mathrm{eV}$ and $\delta_{\text {II }} \approx 2 E_{F} / N_{\text {II }}=230 \mu \mathrm{eV}$. This is in good agreement with the estimates obtained from the $I-V$ curves. From standard measurements we obtain the charging energies $E$ for adding an electron to the dot [11]: $E_{\mathrm{I}}=1.1 \mathrm{meV}$ (dot I) and $E_{\mathrm{II}}=1.8 \mathrm{meV}(\operatorname{dot} \mathrm{II})$.

Transferring one electron through the double dot requires three consecutive tunnel events. The electron has to overcome the charging energy for adding an electron to dot I and dot II. Furthermore, electrostatic interactions between the dots can change the transport conditions. These effects have been studied in detail in metal [11] and semiconductor systems $[12,13]$ in the regime where charging effects dominate transport. We find similar effects, but in this paper we focus on the role of the OD states on tunneling through the double dot and consider the charging energies as constant offsets in the transport conditions.

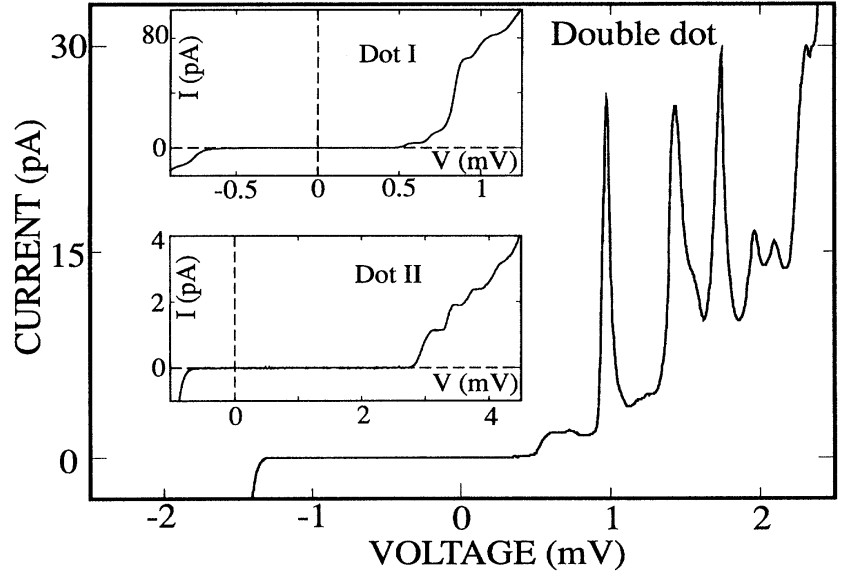

FIG. 2. I-V curve of the double dot, showing sharp resonances in the current when two OD states line up. Upper inset: $I-V$ curve of dot I. Lower inset: $I-V$ curve of dot II. Both insets show a suppression of the current at low voltages due to the Coulomb blockade and a stepwise increase of the current due to the discrete energy spectrum of the dot.

Figure 2 shows the $I$ - $V$ curve of the double dot with all three tunnel barriers set in the tunneling regime. The Coulomb blockade suppresses the current through the double dot at low bias voltages, but at larger bias voltages the current shows sharp resonances. The spacing of the resonances is about $250 \mu \mathrm{eV}$. This is of the same order as the spacing of the OD states in the single dots, which demonstrates that the resonances originate from the discrete energy spectrum in the two dots.

The same resonances are seen when we sweep the gate voltage. Figure 3 shows the current through the double dot versus the voltage $V_{g 1}$ on gate 1 using a constant bias voltage of $280 \mu \mathrm{V}$. The current shows three groups of

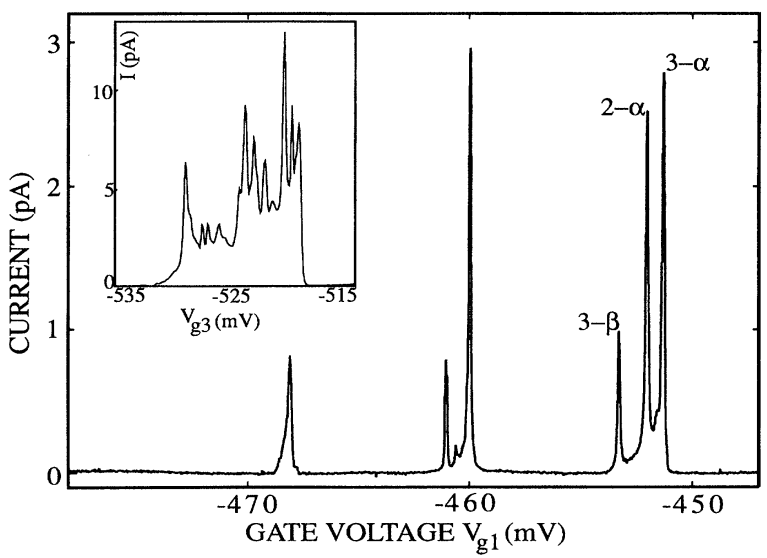

FIG. 3. Current through the double dot vs the gate voltage $V_{g 1}$ using a bias voltage $V=280 \mu \mathrm{V}$. Inset: The current through the double dot as a function of $V_{g 3}$ with $V=1 \mathrm{mV}$ showing that the number of resonances increases with bias voltage. 
sharp resonances separated by regions of zero current with a period $\Delta V_{g 1}=9 \mathrm{mV}$ in gate voltage $V_{g 1}$. With only dot I formed, we observe periodic Coulomb oscillations as a function of $V_{g 1}$ with the same period $\Delta V_{g 1}$; each period corresponds to a change of one electron in dot I. This indicates for the double dot experiment of Fig. 3 that tuning the gate voltage by $\Delta V_{g 1}$ alters the number of electrons $N_{\mathrm{I}}$ by one, while keeping the number of electrons $N_{\text {II }}$ constant.

We now argue that the sharp resonances in the current occur when two 0D states in the two dots are on resonance. Resonant tunneling through the double dot is illustrated in the schematic potential landscape of the double dot in Fig. 1(b). This figure shows a few of the OD states in $\operatorname{dot}$ I (levels 1 to 5) and dot II (levels $\alpha$ and $\beta$ ). The electrostatic potentials $\varphi_{\mathrm{I}}$ and $\varphi_{\text {II }}$ are tuned in such a way that transport through the double dot is possible via the charge states $\left(N_{\mathrm{I}}, N_{\mathrm{II}}\right) \rightarrow\left(N_{\mathrm{I}}+1, N_{\mathrm{II}}\right) \rightarrow\left(N_{\mathrm{I}}, N_{\mathrm{II}}+\right.$ $1) \rightarrow\left(N_{\mathrm{I}}, N_{\mathrm{II}}\right)$. The finite bias voltage $V$ gives an electron from the left reservoir three choices to tunnels into dot I: It can tunnel to one of the unoccupied levels 5,4 , or 3 . This increases the electrostatic potential $\varphi_{\mathrm{I}}$ by the charging energy $E_{\mathrm{I}}$ (the levels have been drawn at the positions applicable after an electron has occupied one of the levels 5,4 , or 3 ). When the incoming electron relaxes to the ground state (level 3), it can tunnel via the levels 3 and $\alpha$ to the right reservoir. When elastic tunnel processes are the dominant transport mechanism, the current through the double dot is resonantly enhanced only when the two OD states in dot I and dot II match in energy. Tuning the alignment of the OD states with the bias voltage $V$ or the gate voltage $V_{g 1}$ gives rise to the sharp resonances in Figs. 2 and 3.

The resonances in a particular group in Fig. 3 can be identified with the energy diagram of Fig. 1(b). The first resonance occurs when level 3 resonates with level $\alpha$ (peak 3- $\alpha$ ). This corresponds to the rightmost peak in Fig. 3. Increasing $\varphi_{\mathrm{I}}$ by making $V_{g 1}$ more negative brings transport off resonance until level 2 lines up with $\alpha$ (peak 2- $\alpha$ in Fig. 3) followed by the third peak 3- $\beta$. Continuing to sweep $V_{g 1}$ increases the energy of level 3 above the electrochemical potential $\mu_{\text {left }}$ of the left reservoir. This blocks transport and removes an electron from dot I permanently. The next group of resonances is observed when the gate voltage $V_{g 1}$ has been changed by one Coulomb oscillation period $\Delta V_{g 1}$ (see Fig. 3). Note that the number of resonances decreases in the next two groups. Sweeping $V_{g 1}$ also shifts the OD states in dot II due to a small capacitance between gate I and dot II [see Fig. 1(a)]. Transport is possible until OD state $\alpha$ is shifted above $\mu_{\text {left }}$ and one electron is removed from dot II permanently $\left(V_{g 1}<-470 \mathrm{mV}\right)$.

The spacing of the OD states can be obtained by converting the gate voltage scale into energy [10]. This yields an energy separation of resonances $2-\alpha$ and 3- $\alpha$ by $70 \mu \mathrm{eV}$, which is the energy separation of levels 2 and 3 .
In the same way we find for levels $\alpha$ and $\beta$ a separation of $200 \mu \mathrm{eV}$. Both values are in good agreement with the typical values we found above.

On increasing the bias voltage we observe that the number of resonances in a particular group increases. This is illustrated by the inset of Fig. 3 which shows one group of resonances with a bias voltage $V=1 \mathrm{mV}$. Approximately 12 resonances are visible in this group as the gate voltage $V_{g 3}$ is swept. These observations are in agreement with the resonant tunneling picture of Fig. 1(b): When the bias voltage is larger, more OD states can cross each other.

Two features of our data justify the above used assumption about relaxation within a dot and allow us to make an estimate for the relaxation rate. First, we do not observe resonances formed by the excited state of the left dot and empty state in the right dot (for instance peak 4- $\alpha$ ). This implies that the relaxation rate of an electron from level 4 to the level 3 is faster than the tunnel rate through the double dot. Second, at relatively small bias voltage $|e V|<\delta_{\mathrm{I}}$, dot I can be only in the ground state and exhibit a single "ground" resonance. At higher bias voltages, it is possible to populate excited states of dot I. When the relaxation rate is much slower than the resonant tunneling rate in the ground state, the system would get stuck in the excited state and the ground resonance would be suppressed. However, we find that the amplitude of this resonance stays constant with increasing bias voltage. From these two observations we deduce that the relaxation rate is faster than the tunnel rate through the double dot. From the maximum current $I_{\max }=10 \mathrm{pA}$ we estimate that relaxation occurs much faster than $e / I_{\max }=2 \mathrm{~ns}$.

We now show that the double dot geometry allows for an accurate measurement of the intrinsic linewidth of the OD states, which is not averaged by the Fermi-Dirac distribution of the electrons in the reservoirs. Theoretical considerations [8] show that the line shape of a resonance is Lorentzian when only elastic tunneling is important. For our geometry, the current reads [8]

$$
I(\varepsilon)=\frac{e}{\hbar} \frac{\Gamma_{3}|t|^{2}}{\varepsilon^{2}+\Gamma_{3}^{2} / 4+|t|^{2}\left(1+\Gamma_{3} / \Gamma_{1}\right)},
$$

where $\varepsilon$ is the energy difference between two discrete states in the two dots and $\Gamma_{1}$ is the linewidth due to tunneling from the left lead to dot I. $t$ is the tunnel coupling between the two dots and $\Gamma_{3}$ is the linewidth due to tunneling from dot II to the right lead. Note that the width of the resonance is determined only by the lifetime of the OD states.

We have studied the line shape in detail in a second sample of identical design. Figure 4 shows a single resonance (black dots). The right-hand side of the peak fits very well with the Lorentzian line shape of Eq. (1) (solid line), while the left-hand side shows a small deviation from the Lorentzian fit. The only free fit parameter is the width at half maximum $w=5 \mu \mathrm{eV}$. From the maximum 


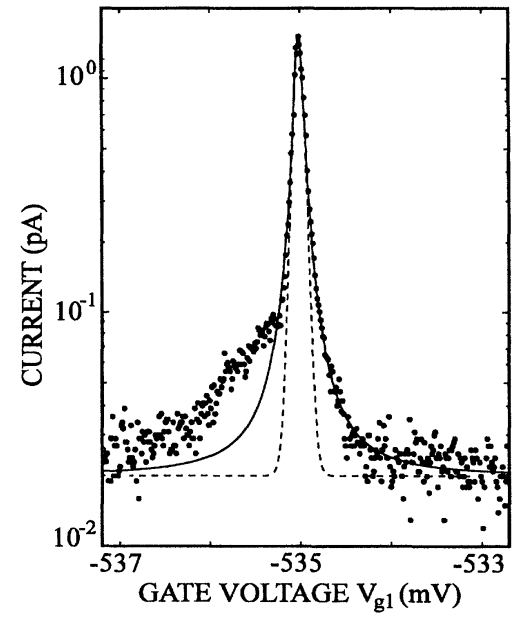

FIG. 4. Enlarged resonance measured in a second device of identical design using a bias voltage of $400 \mu \mathrm{V}$. The data points (black dots) can be fit with a Lorentzian line shape (solid line). For comparison we have plotted a thermally broadened resonance with a fit temperature $T=35 \mathrm{mK}$ (dashed line).

current and the width of the resonance, we find $t \approx$ $0.2 \mu \mathrm{eV}$ and $\Gamma_{3} \approx 10 \mu \mathrm{eV}$. For comparison we have fit the resonance with a thermally broadened resonance $I(\varepsilon) \sim \cosh (\varepsilon / 2 k T)^{-2}$ (dashed line) [14]. The top is fit very well when we use a temperature $T=34 \mathrm{mK}$, but there are large deviations in both tails of the resonance.

We have shown that transport through a double dot is resonantly enhanced when the energies of two OD states match. The width of the resonances is much smaller than the voltage across the double dot. This, together with the Lorentzian line shape of the resonances, demonstrates that transport through the double dot is determined only by the lifetimes of the OD states in the dots. However, we observe a small deviation from the Lorentzian line shape at one side of the resonances. This is a very general feature in our data. It is clearly visible at the high biasvoltage side of the resonances in the $I-V$ curve in Fig. 2 and at the left-hand side of the resonances in Figs. 3 and 4. The asymmetry appears at the side where an electron tunnels from a OD state with a higher energy to a OD state with lower energy. Upon reversing the sign of the bias voltage we find that the asymmetry appears at the other side of the resonance. This provides strong evidence that the asymmetry is due to inelastic tunnel processes. In such a process, an electron can tunnel inelastically upon emitting either a photon or phonon to the environment of the dot. Although we are able to extract the rate of this inelastic process from the data, at present there is no quantitative theory available for our system to allow for a detailed comparison.

In conclusion, we have observed resonant tunneling through two discrete energy states. We showed that resonant tunneling can be used as a new technique to investigate the physics of coupled discrete energy states.

We would like to thank L. J. Geerligs, L. P. Kouwenhoven, and A.T. Johnson for discussions, A. van der Enden and A. van Run for contributions to the device fabrication, and the Delft Institute for MicroElectronics and Submicron Technology for the use of their facilities. Financial support from FOM is gratefully acknowledged.

*Present address: Physikalischen Institut, RWTH-Aachen, Templergraben 55, D-52056 Aachen, Germany.

'Present address: Department of Physics, University of Nottingham, Nottingham NG72RD, United Kingdom.

[1] M. A. Reed et al., Phys. Rev. Lett. 60, 535 (1988).

[2] B. J. van Wees et al., Phys. Rev. Lett. 62, 2523 (1989).

[3] B. Su, V. J. Goldman, and J. E. Cunningham, Science 255, 313 (1992); P. Gueret et al., Phys. Rev. Lett. 68, 1896 (1992).

[4] A. T. Johnson et al., Phys. Rev. Lett. 69, 1592 (1992).

[5] E. B. Foxman et al., Phys. Rev. B 47, 10020 (1993).

[6] R. C. Ashoori et al., Phys. Rev. Lett. 68, 3088 (1992).

[7] B. Meurer, D. Heitmann, and K. Ploog, Phys. Rev. Lett. 68, 1371 (1992).

[8] Y. V. Nazarov, Physica B 189, 57 (1993).

[9] M. Tewordt et al., Appl. Phys. Lett. 60, 595 (1992); U. Shivan et al., Europhys. Lett. 25, 605 (1994).

[10] For a review, see Single Charge Tunneling, edited by H. Grabert and M. H. Devoret (Plenum, New York, 1992). For a collection of quantum dot papers, see The Physics of Few Electron Nanostructures, edited by L. J. Geerligs et al. [Physica B 189 (1993)].

[11] H. Pothier et al., Europhys. Lett. 17, 249 (1992).

[12] C. J. B. Ford et al., Nanostructured Mater. 3, 283 (1994); M. Kemerinck and L. W. Molenkamp, Appl. Phys. Lett. 65, 8 (1994); I. M. Ruzin et al., Phys. Rev. B 45, 13469 (1992).

[13] R. H. Blick et al. (to be published); F. R. Waugh et al. (to be published).

[14] C. W. J. Beenakker, Phys. Rev. B 44, 1646 (1991). 

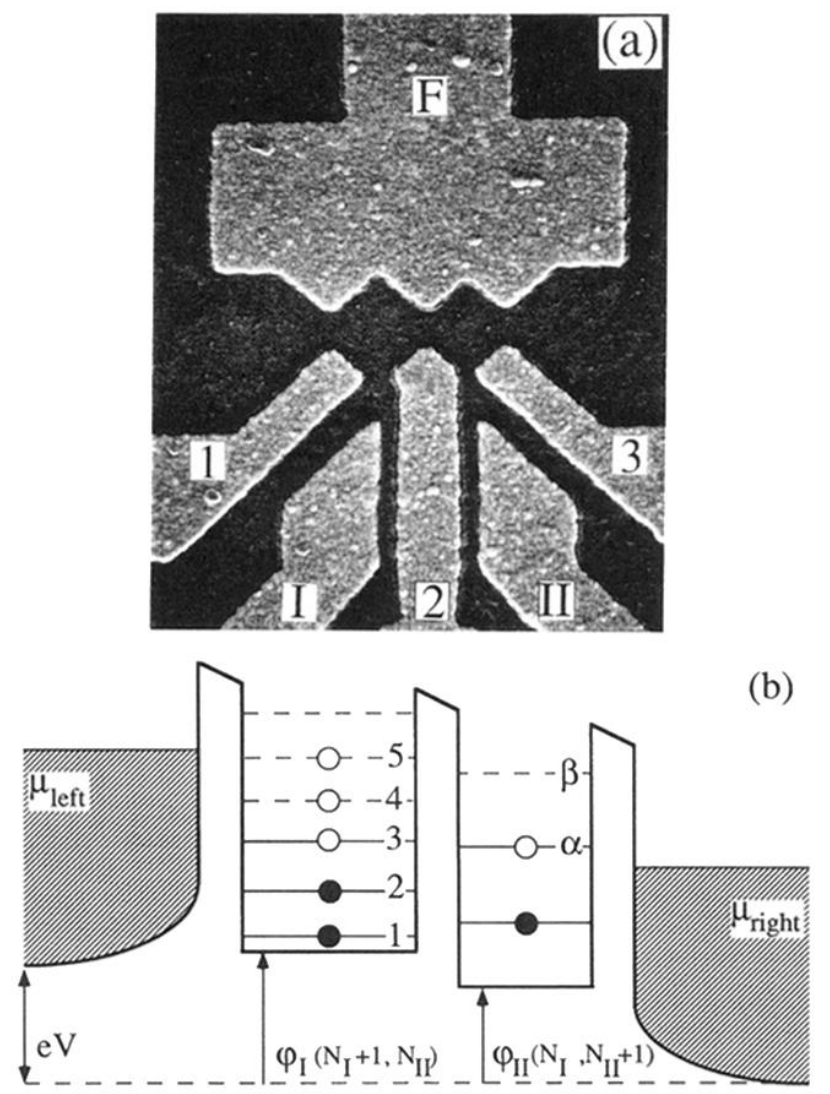

FIG. 1. (a) SEM micrograph of the double dot with lithographic dimensions of $320 \times 320 \mathrm{~nm}^{2}$ (left dot) and $280 \times$ $280 \mathrm{~nm}^{2}$ (right dot). (b) Schematic potential landscape of the double quantum dot, where $\mu_{\text {left }}$ and $\mu_{\text {right }}$ denote the electrochemical potentials of the left and right reservoirs and $V$ the bias voltage across the double dot. The 0D states in dot I are denoted by levels 1 to 5 and in dot II by levels $\alpha$ and $\beta$. 